\title{
Application of Gelatin Derived from Waste Tilapia Scales to an Antibiotic Hydrogel Pad
}

\author{
Benjamaporn Wonganu ${ }^{1, *}$ \\ ${ }^{1}$ Department of Biotechnology, Faculty of applied Science, King Mongkut's University of Technology North Bangkok, Bangkok, \\ Thailand
}

\begin{abstract}
The main purpose of this research is to optimize the process to change useless fish scales to be a hydrogel that can be used as drug-loaded materials. The results of morphological structure of the extracted gelatin powders derived from Tilapia scales using lime juice as natural acid in the extraction process showed appropriated like-polymer structure. The diffractogram pattern acquired on the pure gelatin powder were typical of a partially crystalline gelatin with a sharp peak with low intensity located at $2 \theta=\sim 7^{\circ}$ and a broad peak located at $2 \theta=\sim 19^{\circ}$. This data can be confirmed the structure of the triple-helical crystalline structure in gelatin in the extracts. Chemical bonding analysis using FTIR revealed no difference in structure bonding between gelatin extracted from using synthetic acid and using natural acid. The hydrogel pads were fabricated based on using gelatin powder extracted from tilapia scales with natural acid and using CMC as cross-linking substance. This formulated hydrogel pad hold $10 \%-30 \%(\mathrm{v} / \mathrm{w})$ of Thai herbal plai formula for pain and inflammatory treatment. The percent volume of Thai herbal plai was calculated to weight of extracted fish gelatin powder that was used in the hydrogel formulation. The maximum of $30 \%(\mathrm{v} / \mathrm{w}) \mathrm{can}$ be added into the hydrogel pad and it showed the inhibition of Escherichia coli growth. This natural hydrogel could be a promising candidate to be used as natural medical gel bandages for people who have an allergy to general paper or plastic bandages.
\end{abstract}

\section{Introduction}

Gelatin is one material that is general used in various applications such as providing gelling ingredient in cosmetic and health care products, using for encapsulation of drugs in pharmaceutical industries or using as stabilizers, thickeners, emulsifiers, foaming agents, water binder, and crystal growth modifier in food industries [13]. Gelatin is derived from partial hydrolysis of fibrous structure of collagen. Collagen and gelatin are commonly found in bovine hide, pig skin or chicken waste, however, these sources have faced some constrains related to biological contaminants and religious issues [4]. Seafood processing wastes such as skin, bones, scales and fins are alternative sources for collagen and gelatin extraction. Fish skin and bones have been major parts for collagen extraction.

To prepare fish gelatin, the triple helix structure of extracted collagen is broken down in the hydrogen and covalent bonds, resulting in helix-to-coil transition and conversion into soluble gelatin. The source of collagen and the processing conditions will influence the physical properties of the gelatin. It might be modified to use as a component of biopolymers $[5,6]$. Fish scales are an interesting fish by-product that is an abundant resource generally found in any fresh market in Thailand. Waste from fish scale is an abundant source that could be reversed to a value-added product for various applications. The research focused specifically in Tilapia scales because Tilapia nilotica Linn is an economic important freshwater fish. Thailand is in the fourth ranking of the world's fresh water fish exporting country. Therefore, huge amounts of fish wastes are discarded in the processes every day.

Interestingly, $17.00 \pm 1.15 \%$ of collagen could be obtained from Tilapia scales, so this type of waste could be potential source for gelatin extraction [7]. This research focused on study of properties of obtained gelatin powder derived from waste tilapia scales and its application in a natural medical gel pad. Thai herbal plai formula for pain and inflammatory treatment is supported from Niraco Skincare, a SME company in Thailand. Niraco Skincare developed this herbal medicine as a commercial product. Besides, phenylbutanoids in the crude extract of Zingiber cassumunar Roxb revealed high antibacterial activity against Staphylococcus aureus and Escherichia coli [8]. Thus, Thai herbal plai was selected as a candidate loading drug in the hydrogel pad formulated with fish scale gelatin. Application of fish scale wastes to generate a naturally medical material might be a potential role in recycling of waste.

\section{Materials and methods}

\subsection{Materials}

Fresh Tilapia (Oreochromis niloticus) scales were obtained from the local fresh market in Nonthaburi province. Acetic acid, sodium hydroxide, sodium chloride, EDTA, isobutyl alcohol and other reagents in

\footnotetext{
${ }^{*}$ Corresponding author: Benjamaporn.w@sci.kmutnb.ac.th
} 
cell culture were all of analytical grade and purchased from HiMedia, India and Sigma, USA. Thai herbal plai used as a candidate of a medical Thai herb are supported from Niraco Skincare Thailand. Escherichia coli (ATCC 25922) were supported from National Nanotechnology Center, National Science and Technology Development Agency Thailand.

\subsection{Gelatin yield}

The yield of gelatin was calculated based on dry weight of fish scale by using the following equation:

$$
\text { Yield of gelatin }(\%)=\frac{\text { weight of dried gelatin }(g)}{\text { Dry weight of fish scale }(g)} \times 100
$$

\subsection{FTIR spectroscopy}

Fourier transform infrared (FT-IR) spectroscopy of gelatin sample was performed using an FT-IR spectrophotometer (PerkinElmer spectrum version spectrum 2000)

\subsection{Scanning electron microscopy (SEM) analysis and Energy Dispersive Spectrometry (EDS)}

The surface morphology of gelatin was analyzed by scanning electron microscope (HITACHI SU-5000, Germany). The surface of dried gelatin was coated with gold in vacuum using sputter coater, and was photographed. The chemical compositions in gelatin powder were determined by EDS.

\subsection{The process of gelatin extraction from waste Tilapia scale}

The processes of gelation preparation were adapted from Paul Das and Prasad method (2017) [9]. 30 g of Tilapia fish scales had been washed and overlaid in the oven until they were completely dried. They were soaked with $5 \%$ $\mathrm{NaCl}(1: 10, \mathrm{w} / \mathrm{v})$ for $30 \mathrm{~min}$ twice to make fish scales swell. After discarding $\mathrm{NaCl}$, they were soaked $0.4 \%$ $\mathrm{NaOH}(1: 10, \mathrm{w} / \mathrm{v})$ and stirring for $30 \mathrm{~min}$ twice to remove any contamination from fish collagen. The samples were in $10 \%$ isobutyl alcohol $(1: 4, \mathrm{w} / \mathrm{v})$ and stirring for $30 \mathrm{~min}$ three times to remove fat. $0.25 \mathrm{M}$ of EDTA pH $7.66(1: 2$, $\mathrm{w} / \mathrm{v}$ ) was used to change the condition of the samples to be neutral. The solution was changed every $12 \mathrm{~h}, 2 \mathrm{~h}, 2 \mathrm{~h}$ and $1 \mathrm{hr}$, respectively. The samples were hydrolyzed with acids. The experiment compared between lime juice $(1: 3$, $\mathrm{w} / \mathrm{v})$ for $24 \mathrm{~h}$ and acetic acid for $3 \mathrm{~h}$ The samples were washed with distilled water several times and soaked in distilled water $(1: 3, \mathrm{w} / \mathrm{v})$ at $60^{\circ} \mathrm{C}$ for $12 \mathrm{~h}$ The supernatant was collected and poured in plastic tray as a thin layer and leave at room temperature for $12 \mathrm{~h}$ The samples were homogenized and kept as the gelatin powder in refrigerator.

\subsection{Wide-angle X-ray diffraction (WAXD) analysis of gelatin}

Characterization of gelatin powder was done using wideangle X-ray diffraction technique. It was carried out in an X-ray diffractometer using 4-Tesla superconducting wavelength shitter as radiation source and operated at 8 $\mathrm{keV}$ voltages and $100 \mathrm{~mA}$ current. The pattern was recorded in a $\theta-2 \theta$ configuration.

\subsection{The fabrication of hydrogel pad containing Thai herbal plai}

The 36 formula of Hydrogel pad formation containing Thai herbal plai were developed. The processes were adapted to be used in varied concentration of glycerol as a plasticizer, and carboxyl methyl cellulose (CMC) as a cross-linking substance. $8 \% \mathrm{w} / \mathrm{v}$ of extracted fish gelatin was mixed with varied concentration of glycerol (20-40\% $\mathrm{v} / \mathrm{v})$ and varied concentration of CMC $(20-40 \% \mathrm{w} / \mathrm{v})$. Thai herbal plai in different concentrations $(10-30 \% \mathrm{v} / \mathrm{w}$ of gelatin powder) were varied in each formula of hydrogel forming to figure out the best formula to get a good physical hydrogel pad. $25 \%$ of Tween 80 was used as a surfactant in the experiment. The mixture was blended in the hot plate for $3 \mathrm{~min}$. The casting was performed in a $100 \times 15 \mathrm{~mm}$ glass plate. The gel was dried in $40^{\circ} \mathrm{C}$ oven for $15 \mathrm{~h}$ Then, the gel was set in room temperature for $24 \mathrm{~h}$

\subsection{The Disk/Gel diffusion antibiotic sensitivity test (The Kirby-Bauer test)}

E. coli ATCC 25922 was grown in Nutrient Broth (NB) and incubated in shaking incubator at $37^{\circ} \mathrm{C}, 150 \mathrm{rpm}$ for $24 \mathrm{~h}$ Then, $5 \times 10^{7}$ cells $/ \mathrm{ml}$ of $E$. coli was used in the Kirby-Bauer test. The area of $0.569 \mathrm{~mm}^{2}$ of the hydrogel containing Thai herbal plai was put on each culture plate. The inhibition zone was measured after the culture plate was incubated at $37^{\circ} \mathrm{C}$ in incubator for $24 \mathrm{~h}$ The hydrogel containing Ampicillin was used as a positive control and the hydrogel without Thai herbal plai was used as a negative control.

\section{Results and discussions}

\subsection{Percent yield of different hydrolysis acids}

Different acids to hydrolyse and to extract gelatin powder from $1 \mathrm{~g}$ of fish scale showed different percent yields. The results showed that using $0.05 \mathrm{M}$ acetic acid $(\mathrm{pH} 2.4)$ provided $4.6 \pm 1.7 \%$ dry weight of gelatin powder yields, whereas using lime juice ( $\mathrm{pH} 3.2$ ) obtained $15.7 \pm 0.2 \%$ dry weights. It can suggest that Lime juice can be used as natural hydrolysis acid for gelatin extraction. Lime juice contains weak tricarboxylic acid called citric acid in the concentration of $1.38 \mathrm{~g} / \mathrm{oz}$ or $0.0475 \mathrm{~g} / \mathrm{mL}$ [10].

In the experiment of gelatin extraction, $1 \mathrm{~g}$ of fish scales was incubated in $3 \mathrm{~mL}$ of lime juice $(0.143 \mathrm{~g}$ citric acid. Gelatin powder derived from lime juice hydrolysis provided more satisfied odor, although, the incubation time in using lime juice took longer than of using $0.05 \mathrm{M}$ acetic acid. Lime juice helps to eliminate critical problem 
in an unsatisfied odor of fish gelatin that affects food application.

\subsection{Characterization of biopolymer}

The chemical stability of gelatin has an influence on their applications. Degradation like partial oxidation is concerned. FTIR measurement was a technique to identify the possible biomolecules, chemical bonds responsible for the structural and functional stabilization of the gelatin extracted from the fish scales. Amide bands in the polypeptide and protein represent different vibrational modes of the peptide bond. The results of absorption bands of gelatin from two different processes in the IR spectra were similar. The results (Fig. 1) showed the peaks of the gelatin at $3300-3500 \mathrm{~cm}^{-1}$ attributed to the presence of hydrogen bond water and amide-A. $1630 \mathrm{~cm}^{-1}$ peaks corresponded to the occurrence of amide-I, at $1565 \mathrm{~cm}^{-}$ ${ }^{1}$ and $1240 \mathrm{~cm}^{-1}$ indicated amide-II and the amide-III, respectively (9).

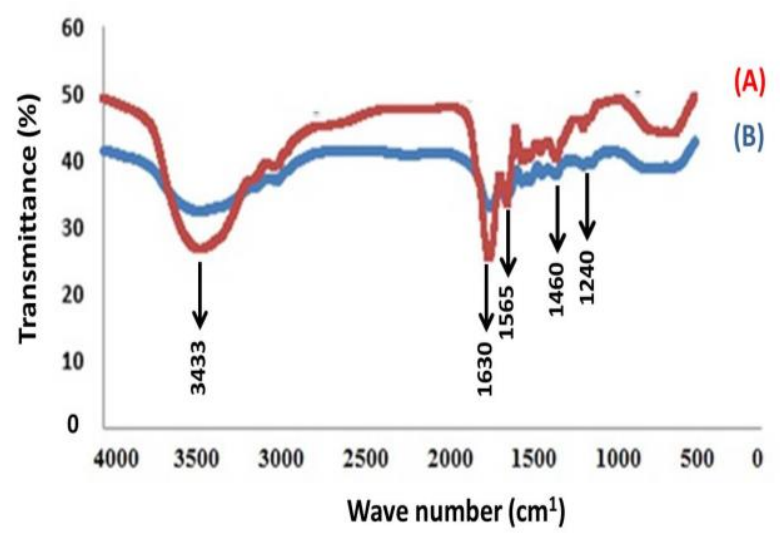

Fig. 1. FTIR spectra of gelatin powder derived from different processes; using lime juice in hydrolysis process (A) and using $0.05 \mathrm{M}$ acetic acid in hydrolysis process (B).

The microstructures of obtained gelatin powder from two processes were examined by Scanning Electron Microscopy (SEM) and compared with the commercial gelatin. The pore structure determined by SEM provides the information on surface related to biological properties such as permeability. Porosity can be used as a tool to predict drug release in biomaterial application [11]. Rough surface with hollow cells were observed from gelatin derived from using lime acid hydrolysis (Fig. 2C). Whereas, commercial gelatin and gelatin derived from using chemical acid showed small rough flake (Fig. 2A and Fig. 2B, respectively). Gelatin powder made from lime hydrolysis process might provide a favorable biological environment for some applications, especially in biomaterial sciences.

The diffractogram pattern form WAXD revealed a sharp peak with low intensity located at $2 \theta=\sim 7^{\circ}$ and a broad peak located at $2 \theta=\sim 19^{\circ}$ as typical of a partially crystalline gelatin (Fig. 3). These obtained peaks were characterized to the triple helical crystalline structure in gelatin $[12,13]$

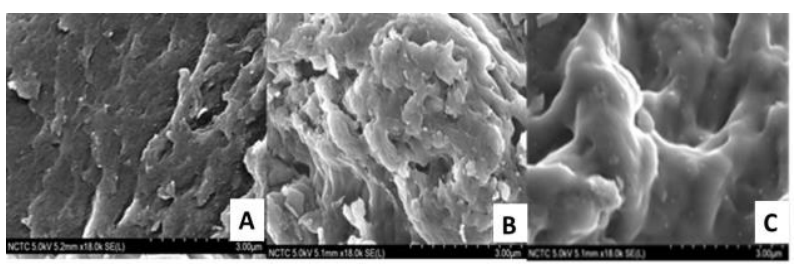

Fig. 2. SEM micrograph of gelatin extracted from different source; (A) commercial gelatin powder, (B) gelatin powder hydrolyzed by $0.5 \mathrm{M}$ acetic acid and $(\mathrm{C})$ gelatin powder obtained from using lime acid hydrolysis

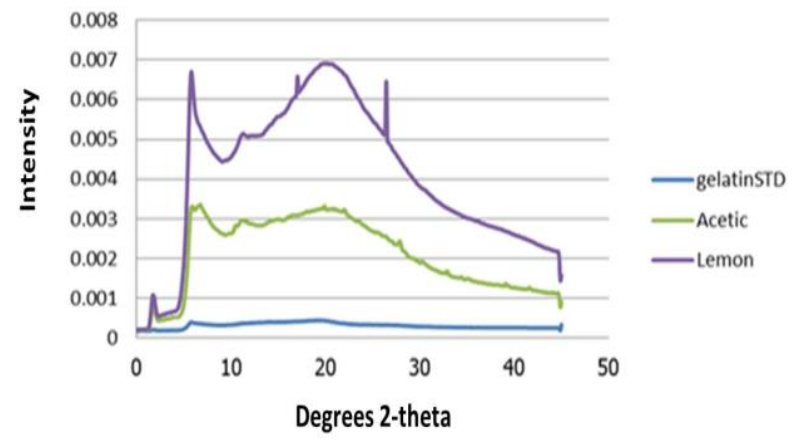

Fig. 3. WAXD pattern of gelatin from different sources; blue line is spectrum peak of commercial gelatin, green line is gelatin made from using acetic acid for hydrolysis and purple line show spectra of gelatin derived from hydrolysis by using lime acid

\subsection{Formulation of hydrogel pad derived from extracted fish scale}

A plasticizer is a chemical substance used to decrease melting point of polymer. It helps to support flexibility and softness of polymers. There were studies reported that a plasticizer helped to form flexible and soft chitosan films $[14,15]$. However, the formula in hydrogel formation processed in the experiment that used only glycerol obtained an unsuitable gel pad for attaching in skin. The gel lacked stability in skin attachment. Carboxyl methyl cellulose (CMC) provided as a cross-linking substance to increase stability in hydrogel formation. The results showed that the formula that applied $30 \%(\mathrm{v} / \mathrm{v})$ glycerol, 30\% (w/v) CMC and $10 \%(\mathrm{v} / \mathrm{w})$ Thai herbal plai was the best one from 36 varied formulas. It can form flexible, soft hydrogel and can set to stable pad forming to attach skin as shown in Fig. 4. Increase of volume of Thai herbal plai in the hydrogel more than $10 \%$ decreased stability in the hydrogel pad setting and attachment to skin.

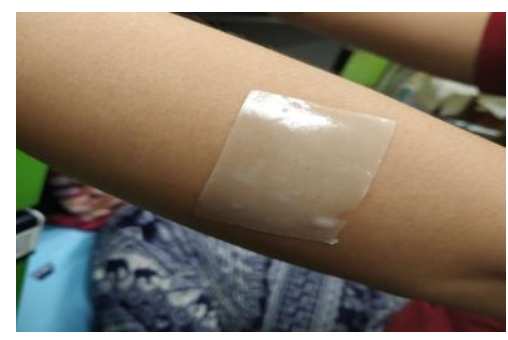

Fig. 4. Skin attachment ability of the hydrogel containing Thai herbal plai 


\subsection{Antibacterial inhibition zone}

The agar disc diffusion method is a cheap, reproducible and practical method to determine the antimicrobial activities. The inhibition zone is measured from a circular area that bacteria colonies do not grow. This zone appears around the spot of the antibiotic $[16,17]$. The Thai herbal plai had antimicrobial activity against $E$. coli as the results showed in the Fig. 5A. Thai herbal plai diffused from paper disc and showed the inhibition zone as labeled with black lines of Fig. 5A. Whereas, melting of gel disc occurred after $37^{\circ} \mathrm{C}$ incubation. The diameter of zone without bacterial growth was measured. The inhibition zones of gel disc test were labelled with the red lines in the figure 5B. The inhibition zones of experiment were subtracted with the clear zone of a negative control. The results showed that the gel disc containing Thai herbal plai 30\%, 20\% and $10 \%(\mathrm{v} / \mathrm{w})$ showed inhibition zone bigger than the gel disc without Thai herbal plai (Figure 5B).

Another method for antibiotic sensitivity test such as dilution broth susceptibility assay is determined as another option to eliminate gel disc melting problem [18]. Nevertheless, it cannot determine the ability to penetrate the medical drug from the hydrogel. The inhibition area of the hydrogel applied in agar plate test was only 0.569 $\mathrm{mm}^{2}$, whereas the whole area of the hydrogel containing $10 \%-30 \%$ Thai herbal plai was $7,857.143 \mathrm{~mm}^{2}$. It means that the drug concentration was decreased 10,000 times. Here, there was an attempt to increase the volume of Thai herbal plai in the hydrogel, however, the hydrogel cannot set after uploading the Thai herbal plai in the hydrogel more than $30 \%$ (Fig. 4 ).

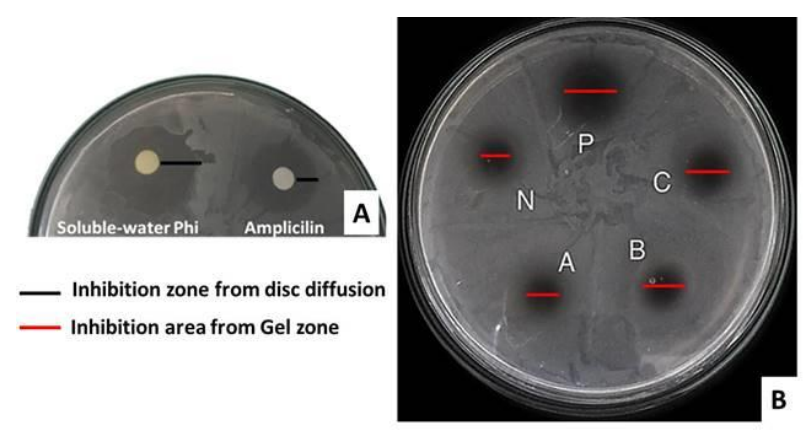

Fig. 5. Inhibition zone from disc diffusion showed antibacterial activity of Thai herbal plai compared with positive control (A), by using plai extract and ampicillin drug. (B) The inhibition zone of hydrogel disc with Thai herbal plai. $\mathrm{P}=$ the hydrogel with ampicillin, $\mathrm{N}=$ the hydrogel without Thai herbal plai, $\mathrm{A}=$ the hydrogel with $10 \%$ Thai herbal plai $(\mathrm{v} / \mathrm{w}), \mathrm{B}=$ the hydrogel with $20 \%$ Thai herbal plai $(\mathrm{v} / \mathrm{w})$ and $\mathrm{C}=$ the hydrogel with $30 \%$ Thai herbal plai (v/w). Dark lines showed inhibition the zone from disc diffusion. Red lines showed the inhibition area from gel zones.

\section{Conclusions}

The utility of waste fish scales to produce gelatin powder and to generate a naturally medical bandage was a main purpose of this research. It helps to increase value of foodprocessing waste for production of high value added material for drug-loading. Physical properties of obtained gelatin might be important for further works, such as specific-molecule linking applications. Although, the efficiency of drug release and loading ability of this hydrogel is still a controversial issue. However, our research is on-going process on development of nanoparticles to incorporate inside this natural hydrogel. Moreover, production of gelatin made from this work using lime acid in the process helps to reduce the odor problem of fish gelatin and increases demand in using fish gelatin to apply as biomaterials for food, pharmaceutical and cosmetic fields. Besides, current trends of natural product, this natural fish hydrogel might be a prototype for a natural material applied for medical and cosmetic field with non-toxic in animal cells. The example of applications fish gelatin is shown in the synthesis of gelatin methacryloyl (GelMA), biomaterial used in various biomedical fields. It was also synthesized by using fish-derived gelatin to avoid a risk of transmission of diseases, such as mad cow disease, as well as certain religious restrictions. Therefore, recycling fish waste scales not only benefits to be value added products to protect the environment, but also it might gain a value for the health and safety of the population.

The research is supported by department of biotechnology, faculty of applied science, KMUTNB. We thank for Dr. Tanyakorn Muangpoh, National Nanotechnology Center, National Science and Technology Development Agency Thailand and Synchrotron Light Research Institute for assistance with research equipment.

\section{References}

1. S. Sultana, M.E. Ali, M.N.U. Ahamad, Preparation and Processing of Religious and Cultural Foods (2018), pp. 215-239.

2. S. H. Flôres et al., Reference Module in Food Science (2017).

3. I.J. Haug, K.I. Draget, Handbook of Food Proteins (2011), pp. 92-115.

4. A. Aberoumand, World J Fish Mar. Sci. 2, 204 (2010).

5. M. Koliada, V. Plavan, C. W, V. Barsukov, (5th International conference on advanced materials and systems (ICAMS)) (2014).

6. M.C. Gómez-Guillén, B. Giménez, M.E. LópezCaballero, M.P. Montero, Food Hydrocoll. 25, 1813 (2011).

7. S. Morimura et al., Process Biochem. 37, 1403 (2002).

8. S.S. Taechowisan, W.S. Phutdhawong, J Appl Pharm Sci. 8, 121 (2018).

9. M. Paul Das, S.P. R, K. Prasad, V. Jv, R. M, Biopolymer 9, 239 (2017)

10. K.L. Penniston, S.Y. Nakada, R.P. Holmes, D.G. Assimos, J Endourol. 22, 567 (2008).

11. I.S. Raja, N.N. Fathima, Springerplus 3, 2193 (2014).

12. I. Yakimets et al., Polymer. 46, 12577 (2005). 
13. C. Peña, K. de la Caba, A. Eceiza, R. Ruseckaite, I. Mondragon, Bioresour Technol. 101, 6836 (2010).

14. L. Deng, X. Kang, Y. Liu, F. Feng, H. Zhang, Food Hydrocoll. 74, 324 (2018).

15. E.D. Berry, J.E. Wells, Adv Food Nutr Res. 60, 67 (2010).

16. C. Maidment, NFS. 39, 29 (2009).
17. H.S. Bhargav, S.D. Shastri, S.P. Poornav, K.M. Darshan, M.M. Nayak, (2016 IEEE 6th International Conference on Advanced Computing (IACC)). (2016), pp. 409-414.

18. B.Z. Bouhadjera, K.B. Tabti, Pak J Biol Sci. 8, 206 (2005). 